\title{
Acoustothermal Atomization of Water Nanofilms
}

\author{
Rohit Pillai, Matthew K. Borg, and Jason M. Reese \\ School of Engineering, University of Edinburgh, Edinburgh EH9 3FB, United Kingdom
}

(Received 13 February 2018; published 7 September 2018)

\begin{abstract}
We report nonequilibrium molecular simulations of the vibration-induced heating of nanoscale-thick water layers on a metal substrate. In addition to experimentally confirmed acoustothermal evaporation, we observe hitherto unmapped nucleate and film boiling regimes, accompanied by the generation of unprecedented heat fluxes $\left[\sim \mathcal{O}\left(10^{9}\right) \mathrm{W} / \mathrm{m}^{2}\right]$. We develop a universal scaling parameter to classify the heat-transfer regimes and to predict the thickness of the residual nonevaporating liquid layer. The results find broad application to systems involving drying, coatings, and sprays.
\end{abstract}

DOI: 10.1103/PhysRevLett.121.104502

When a solid substrate supporting a film of liquid is vibrated using high-intensity ultrasound [Fig. 1(a)], the liquid can be induced to disintegrate into a fine mist of droplets. This ultrasonic "atomization" of liquids was first discovered in the early 20th century for heavy oils [1]; it has since been demonstrated with liquids of various viscosities and densities [2,3], and is now routinely used in numerous industries [4-6]. However, the current theoretical understanding of atomization phenomena is incomplete at smaller length scales, where thermal effects gain importance. As this Letter will show for the first time using highfidelity molecular simulations, vibration-induced heating can be used to evaporate or boil a water nanofilm, and the heat fluxes observed in the process are higher than any previously reported values. This is broadly relevant to applications involving acoustofluidics, thin-film heating, and surface-driven phase change.

The experimental study of atomization is challenging, as acoustic effects occur at a shorter time scale, $t_{a}=1 / f$, when compared to the hydrodynamics, $t_{h}=\mu h_{0} / \gamma$, where $f$ is the ultrasonic frequency, $\mu$ is the liquid viscosity, $h_{0}$ is the initial height of the film, and $\gamma$ is the interfacial tension of the free surface. For a macroscopic water film of height $h_{0}=10^{-3} \mathrm{~m}$, however, the ratio of time scales is large $\left[t_{R}=t_{h} / t_{a} \sim \mathcal{O}\left(10^{2}\right)\right]$, assuming the megahertz-order frequencies commonly employed in atomization applications. Consequently, the underlying physical mechanisms have been deduced by visual examination of high-speed camera images, and measurements of atomized drop velocities and size distributions [7-9]. The consensus is that atomization results from a conjunction of unstable

Published by the American Physical Society under the terms of the Creative Commons Attribution 4.0 International license. Further distribution of this work must maintain attribution to the author(s) and the published article's title, journal citation, and DOI. capillary-wave formation at the liquid interface, along with acoustic-cavitation bubble formation and collapse in the liquid bulk [10,11] [Fig. 1(b)].

Despite the fact that heating of the liquid film due to viscous dissipation is inevitable following the application of ultrasound [12], the phenomenological conjunction theory outlined above disregards vibration-induced heating (or "acoustothermal") effects. This is because the experimentally observed rise in film temperature $\left(T_{f}\right)$ during atomization is typically small at the macroscale $\left(\Delta T_{f}<10 \mathrm{~K}\right.$ [9]), which is not entirely surprising as inertial effects occur only

(a) Application of ultrasound

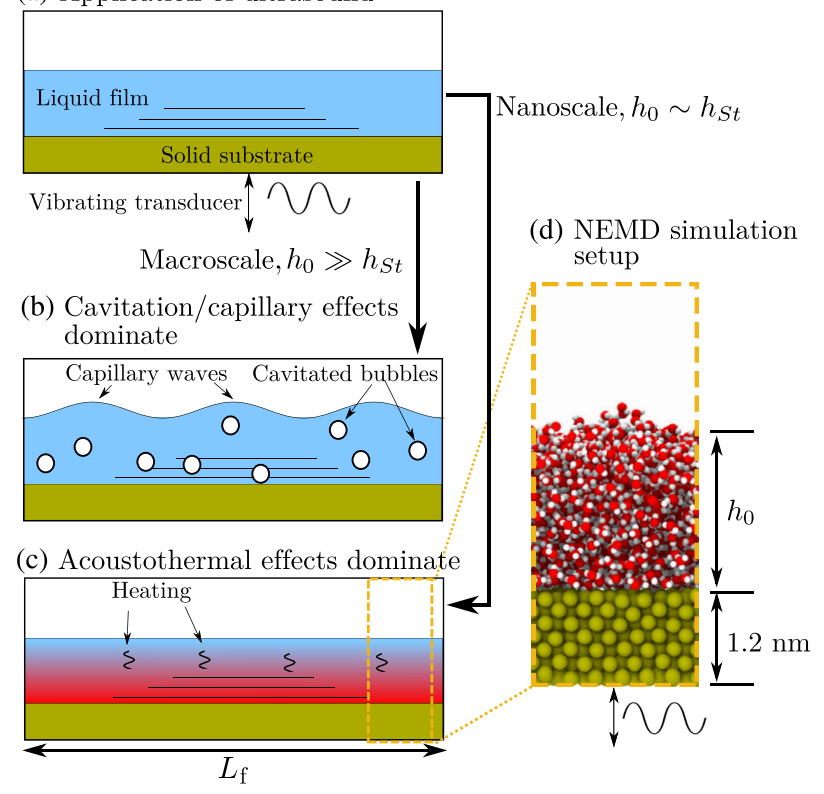

FIG. 1. Schematics of (a) typical ultrasonic atomizer setup, (b) macroscale conjunction theory of atomization, with cavitation inside the fluid and capillary waves at the surface, (c) nanoscale acoustothermal heating and atomization, and (d) the NEMD problem setup for the simulations in this work. 
in a thin Stokes boundary layer of height $h_{\mathrm{St}}=\sqrt{\mu / \rho \omega}$ [13] adjacent to the substrate, where $\rho$ is the liquid density and $\omega=2 \pi f$. Analogous to the time-scale ratio $t_{R}$, a ratio of length scales can be defined as $h_{R}=h_{0} / h_{\mathrm{St}}$; for a film of height $h_{0}=10^{-3} \mathrm{~m}$ vibrated at $f=1 \mathrm{MHz}, h_{R} \sim 10^{3}$, indicating that viscous heating is confined to a negligible region of the macroscale liquid initially. However, acoustothermal heating can be influential in the latter stages of atomization once the film height approaches the microscale and below (i.e., as $h_{R} \rightarrow 1$ ). Recent experimental studies aimed at drying wet fabric by atomizing the contained liquid ultrasonically [14] have observed that surface-capillary waves stop forming on the liquid surface once its volume falls below a critical limit, but the volume continues to decrease. Infrared images showed $\Delta T_{f}=40-50 \mathrm{~K}$, demonstrating that the conjunction mechanisms had given way to acoustothermal evaporation at this point. Acoustothermal effects are also important for applications where $h_{R}$ is initially small, such as micro- or nanofluidic devices using high-frequency surface or bulk acoustic waves [15]. In such systems, significant heating $\left(\Delta T_{f}=50-100 \mathrm{~K}[21,22]\right)$ has been observed, which can be exploited for biological and chemical applications [23,24].

Despite being key to applications as disparate as ultrasonic dryers and lab-on-a-chip devices, micro- or nanofluidic acoustothermal phenomena remain difficult to study experimentally. Some (separate) experiments have shown that focused ultrasonic nebulisers can cause local superheating in liquid fountains $\left(\Delta T_{f}=175-225 \mathrm{~K}[25,26]\right)$, but little theoretical understanding exists of the mechanics or limits of acoustothermal phenomena. The scope of this Letter is to (1) introduce the use of particle-based simulations to investigate acoustothermal effects in liquid nanofilms at high frequencies, and (2) characterize the observed (and hitherto unmapped) acoustothermal evaporation, nucleate boiling, and film boiling regimes.

To the authors' knowledge, this Letter represents the first numerical investigation of acoustic atomization phenomena at any scale; this is a formidable problem due to the lengthscale and time-scale disparities, as well as modeling challenges such as the complex fluid-structure interactions and rapid topographical changes of the free surface. We are able to circumvent the former issue by focusing on nanoscale systems where the acoustic and hydrodynamic scales coincide $\left(t_{R}, h_{R} \sim 1\right)$. We resolve the latter issues by using nonequilibrium molecular dynamics (NEMD), which is the application of classical mechanics to the dynamics of molecular systems, wherein the equations of motion for each molecule are derived from Newton's second law of motion. NEMD has accurately calibrated solid-fluid interaction forces, allows for a simulation time-step much smaller than $t_{a}$, and is well established for problems involving heat transfer in nanofilms.

The simulation domain [shown in Figs. 1(c) and 1(d)], which is periodic in all three directions, contains a liquid

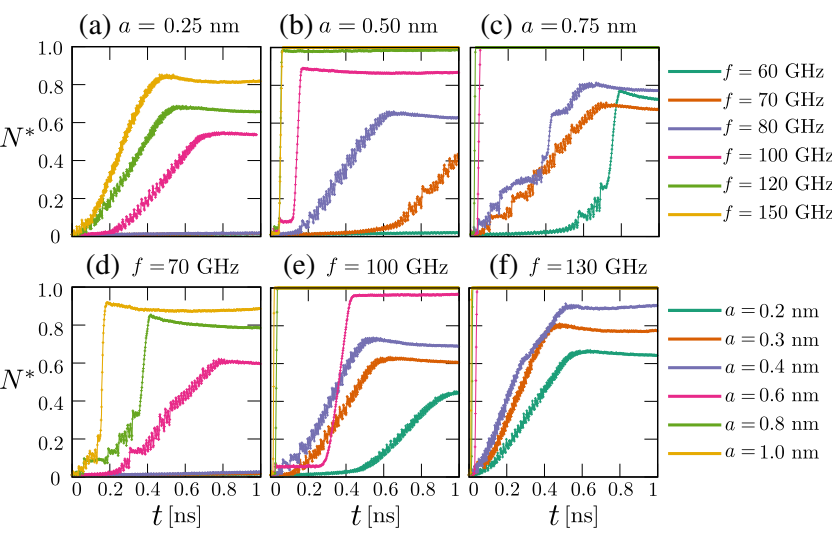

FIG. 2. Time evolution of the normalized atomization $N^{*}$, over a range of vibration frequencies $f$ and three vibration amplitudes (a) $a=0.25 \mathrm{~nm}$, (b) $a=0.50 \mathrm{~nm}$, (c) $a=0.75 \mathrm{~nm}$; and over a range of vibration amplitudes $a$ and three vibration frequencies (d) $f=70 \mathrm{GHz}$, (e) $f=100 \mathrm{GHz}$, (f) $f=130 \mathrm{GHz}$.

nanofilm initialized on a substrate of height $\sim 1.2 \mathrm{~nm}$. Following the applications that motivate this study $[14,23,24]$, we use water as the working fluid and a hydrophilic metal for the vibrating substrate. The substrate and film are initialized at $T_{0}=300 \mathrm{~K}$; while no restriction is placed on the film temperature during the simulation, the substrate is maintained at $300 \mathrm{~K}$ to physically represent a portion of a larger bulk solid that acts as a heat sink. Additional details about the NEMD equations, interaction parameters, and other simulation setup or operation details are provided in the Supplemental Material [27], which includes Refs. [28-33]. In the remainder of this Letter, we focus on discussing our results.

A film of side dimension $L_{f}=11.76 \mathrm{~nm}$ and height $h_{0}=2.17 \mathrm{~nm}$ containing $N_{0}=10000$ water molecules was used as a base case; the effect of varying film dimensions is explored later. The substrate was vertically oscillated at a velocity $\boldsymbol{v}=a \omega \cos (\omega t)$, where $a$ is the oscillation amplitude. A systematic study was performed across a wide range of oscillation frequencies and amplitudes, and the number of atomized molecules, $N_{A}$, was identified using a simple criterion described in the Supplemental Material [27]. The time evolution of the normalized atomization $\left(N^{*}=N_{A} / N_{0}\right)$ for various $f$ and $a=0.25,0.5$, and $0.75 \mathrm{~nm}$ are shown in Figs. 2(a)-2(c), and for various $a$ and $f=70,100$, and $130 \mathrm{GHz}$ are shown in Figs. 2(d)-2(f). Regardless of whether $f$ or $a$ is increased, the observed atomization occurs via three distinct modes; here, we identify the modes using $N^{*}$ (Fig. 2) and classify the cases into regimes I, II, and III in order of increasing vibrational energy. These regimes physically correspond to distinct heat-transfer mechanisms as discussed later. Cases in regime I present as sigmoid curves, with negligible change in $N^{*}$ initially, followed by a region of monotonic increase where $\partial^{2} N^{*} / \partial t^{2}=0$, and a subsequent leveling off to an equilibrium value, $N_{\infty}^{*}\left(0<N_{\infty}^{*}<1\right)$. 

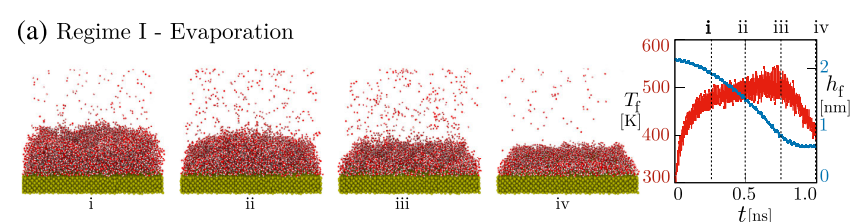

(b) Regime II - Nucleate boiling

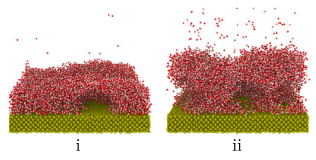

(c) Regime III - Film boiling
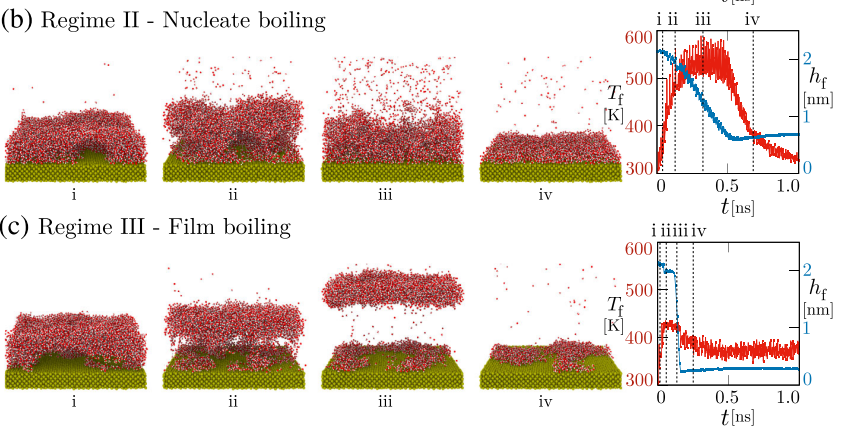

FIG. 3. Time-lapse images (left to center), along with line plots (right) showing evolution of film temperature $T_{f}$ (red) and film height $h_{f}$ (blue), for acoustothermal atomization via (a) evaporation with $a=0.1 \mathrm{~nm}$ and $f=200 \mathrm{GHz}$, at $t=0.25,0.5,0.75$ and $1 \mathrm{~ns}$; (b) nucleate boiling with $a=0.375 \mathrm{~nm}$ and $f=100 \mathrm{GHz}$, at $t=0.06,0.15,0.35$, and $0.7 \mathrm{~ns}$; (c) film boiling with $a=0.5 \mathrm{~nm}$ and $f=100 \mathrm{GHz}$, at $t=0.02,0.05,0.1$, and $0.25 \mathrm{~ns}$.

For cases in regime II, the time lag preceding the onset of atomization is absent, and the equilibrium $N_{\infty}^{*}$ is higher, but is otherwise identical to regime I. Finally, for cases in regime III the atomization is qualitatively different and manifests as one or a series of abrupt jumps in $N^{*}$; also, unlike regimes I and II, all film molecules are atomized $\left(N_{\infty}^{*}=1\right)$ in some cases.

Figures 3(a)-3(c) show snapshots of the atomization process for typical cases in the previously identified regimes I-III, along with line plots showing the evolution of film temperature $T_{f}$ and film height $h_{f}$. Here, $T_{f}$ is calculated using the equipartition theorem,

$$
T_{f}=\frac{1}{N_{f}} \sum_{i=1}^{N_{f}} \frac{2}{3 k_{B}} \sum_{n=1}^{3} \frac{1}{2} m_{i}\left(v_{i, n}-\bar{v}_{n}\right)^{2},
$$

where $N_{f}=N_{0}-N_{A}$ is the number of film molecules, $k_{B}$ is the Boltzmann constant, $m_{i}$ is the mass of molecule $i, v_{i, n}$ is the velocity of molecule $i$ in the $n(=x, y, z)$ direction, and $\bar{v}_{n}$ is the mean flow velocity in the $n$ direction due to the induced oscillations. The time evolution of $T_{f}$ in Fig. 3(a) indicates film superheat $\left(T_{f, \max } \sim 500 \mathrm{~K}\right)$; despite this, images $\mathrm{i}-\mathrm{iv}$ indicate that boiling is absent and the atomization in regime I results from acoustothermal evaporation. Note that the mass flux appears to be highest in Fig. 3(a), iii, which coincides with the peak film temperature, as expected. In contrast to Fig. 3(a), the images in Fig. 3(b) show transient cavitation, i.e., the formation of one [Fig. 3(b), i] or multiple [Fig. 3(b), ii] cavitated bubbles which subsequently collapse [Fig. 3(b), iii]. Regime II thus corresponds to nucleate boiling (see more info in the Supplemental Material [27]), and the maximum film superheat observed $\left(T_{f, \max } \sim 550 \mathrm{~K}\right)$ is close to the experimental limit of 553-583 $\mathrm{K}$ for superheated (metastable) water at atmospheric pressure [34]. Therefore, a first-order phase transition occurs instantly; the radius of the bubbles formed in Fig. 3(b), i and ii are roughly $r_{b} \sim 1 \mathrm{~nm}$. As cavitation is suppressed when $r_{b}$ is smaller than the minimum film dimension [35,36], boiling (transient cavitation) gives way to evaporation (viscous dissipation) in the latter stages of atomization when $r_{b}<h_{f}$ [Fig. 3(b), iii and iv]. Finally, for regime III [Fig. 3(c)], larger bubbles are formed compared to regime II [Figs. 3(c), i, $r_{b} \sim 3-4 \mathrm{~nm}$ ] and, rather than collapsing, they coalesce to form a film of vapor separating the substrate and liquid [Fig. 3(c), ii]. This vapor then drives the film away from the substrate [Figs. 3(c), iii; 3(c), iv], and the insulation it provides also reduces the heat flux into the film, resulting in reduced superheating $\left(T_{f, \text { max }} \sim 410 \mathrm{~K}\right)$; this regime is classified as film boiling [37].

The heat flux into the film $\left(q_{f}\right)$, measured up to the inflection point on the $T_{f}$ curves (see Fig. 3 for examples), can be estimated using $q_{f}=m_{f} c_{p} \Delta T_{f} / L_{f}^{2} \Delta t$, where $c_{p}$ is the specific heat capacity and $\Delta T_{f}=T_{f, \max }-T_{0}$. We obtain values ranging from $q_{f} \sim 10^{8}-10^{9} \mathrm{~W} / \mathrm{m}^{2}$ (regime I) to a maximum of $q_{f, \max } \sim 5 \times 10^{9} \mathrm{~W} / \mathrm{m}^{2}$ (regime II), the latter of which is more than an order of magnitude higher than $q_{f, \max }$ values observed in NEMD studies of thermal boiling [38-41], and close to the theoretical limit achievable in a phase-change process of $q_{f, \max } \sim 2 \times 10^{10} \mathrm{~W} / \mathrm{m}^{2}$ calculated from kinetic theory [42]. While this is consistent with the superheating observed, an interesting question arises: Why would the fluxes generated acoustothermally be higher than corresponding thermally generated values? Note that $q_{f}$ is limited primarily by the onset of film boiling, which reduces the film wetting area and hinders heat transfer. It is well established that the application of ultrasound is effective at delaying (thermal) film boiling, because transient acoustic cavitation prevents the growth and coalescence of bubbles that precede vapor-film formation [43]. The same mechanism, namely, acoustically delayed film boiling, is likely acting here. However, there is one crucial distinction: in our case, both the heat generation within the film as well as enhancement of its transfer occurs acoustothermally.

Figures 3(a), iv and 3(b), iv show that the evaporation process in regimes I and II culminates in a residual nonevaporating layer of thickness $h_{\infty}$ [for Figs. 3(a) and $\left.3(\mathrm{~b}), h_{\infty} \sim 0.65 \mathrm{~nm}\right]$. It is reasonable to assume that the acoustic energy is dissipated isothermally and the system is at equilibrium when $h_{f}=h_{\infty}$. Ignoring the effects of interfacial tension (due to negligible film curvature), the force balance across the liquid-vapor interface at equilibrium simplifies to $P_{f}-P_{v}-P_{D}=0$, where $P_{f}$ and $P_{v}$ are 

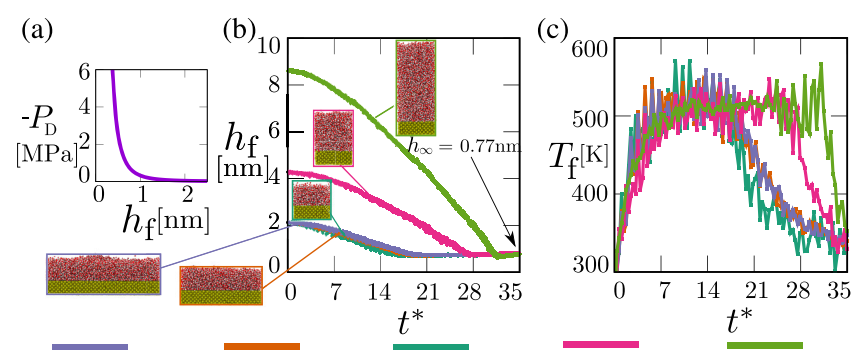

$h_{0}=2.17 \mathrm{~nm}, \quad h_{0}=2.17 \mathrm{~nm}, \quad h_{0}=2.17 \mathrm{~nm}, \quad h_{0}=4.34 \mathrm{~nm}, \quad h_{0}=8.68 \mathrm{~nm}$, $L_{\mathrm{f}}=11.76 \mathrm{~nm} \quad L_{\mathrm{f}}=8.32 \mathrm{~nm} \quad L_{\mathrm{f}}=3.71 \mathrm{~nm} \quad L_{\mathrm{f}}=3.71 \mathrm{~nm} \quad L_{\mathrm{f}}=3.71 \mathrm{~nm}$

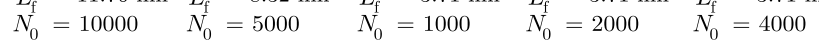

FIG. 4. (a) Variation of the disjoining pressure with film thickness; time evolution of (b) film thickness and (c) film temperature for films of different lengths and heights; $a=$ $0.20 \mathrm{~nm}$ and $f=130 \mathrm{GHz}$ for all cases. Snapshots of films included as insets in (b).

the pressures on the liquid and vapor sides adjacent to the liquid-vapor interface, and $P_{D}$ is the disjoining pressure due to solid-fluid intermolecular forces. While $P_{f}$ and $P_{v}$ depend on acoustic parameters, $P_{D}$, for a given solid-fluid combination, is primarily determined by the film height. The expression for $P_{D}$ in a thin liquid film on a flat surface is $P_{D}=-\Pi / 6 \pi h_{f}^{3}$, where $\Pi$ is the Hamaker constant. In Fig. 4(a) this equation is used to plot $P_{D}$, using $\Pi=$ $5.99 \times 10^{-21} \mathrm{~J}$ [44]. Figure 4(a) indicates that, once $h_{f}<1 \mathrm{~nm}$, the intermolecular forces gain importance and likely suppress evaporation to achieve equilibrium, consistent with NEMD studies of thermal nonevaporating layers [45,46]. An interesting consequence is that $h_{\infty}$ is independent of the initial film dimensions, and depends only on the balance between acoustic and intermolecular forces. This is demonstrated in Fig. 4(b), where the time evolution of $h_{f}$ for fixed $a=0.2 \mathrm{~nm}$ and $f=130 \mathrm{GHz}$ is tracked for films of varying heights $\left(h_{0}\right)$ and lengths $\left(L_{\mathrm{f}}\right)$, and $h_{\infty}=0.77 \mathrm{~nm}$ is observed in all cases. In addition, when scaled by the initial film height $h_{0}$ using $t^{*}=t / t_{h}$, the time evolution of $T_{f}$ is similar for all films [Fig. 4(c)] with divergence only observed once films with lower $h_{0}$ achieve equilibrium, indicating that $q_{f}$ is also independent of film dimensions. This is true for cases in regimes I and II.

While developing an analytical model that fully describes the acoustothermal dynamics in the film is challenging, we note that the force applied by the vibrating substrate scales as $F_{\text {vib }} \sim m_{f} a \omega^{2}$, where $m_{f}$ is the mass of the film. The kinetic energy of these oscillations is converted into internal energy of the water molecules, raising the film temperature; for viscous dissipation this process can be approximated by,

$$
\Phi=\mu\left(\frac{\partial v_{\mathrm{x}}}{\partial y}+\frac{\partial v_{\mathrm{y}}}{\partial x}\right)^{2}
$$

where $\partial v / \partial(x, y)$ are the rate-of-strain components within the film. $\Phi$ thus scales as $\sim \mu\left(v_{\text {ref }} / L_{\text {ref }}\right)^{2}$, where $v_{\text {ref }}$ and $L_{\text {ref }}$ are the characteristic velocity and length scales of the energy
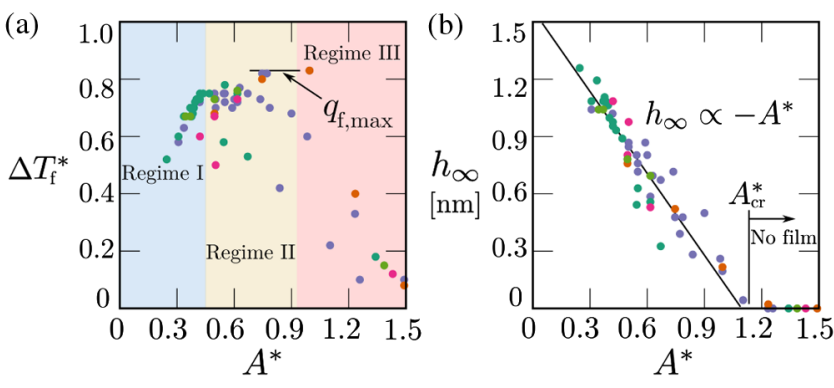

FIG. 5. Plot showing (a) maximum film temperature rise $\left(\Delta T_{\mathrm{f}}^{*}\right)$ and (b) nonevaporating layer thickness $\left(h_{\infty}\right)$ for all our simulations, scaled using the parameter $A^{*}$ of Eq. (3). The same color scheme as in Fig. 4 is used to identify the different films.

dissipation in the fluid. Here, we assume the thermal speed of water as the reference velocity scale $\left(v_{\text {ref }}=\sqrt{k_{B} T_{0} / m_{i}}\right)$, and the Stokes boundary-layer thickness $\left(h_{\mathrm{St}}\right)$ as the reference length scale. A "viscous" force $F_{\mathrm{vis}} \sim m \mu v_{\text {ref }}^{2} t_{a} / L_{\text {ref }}^{3}$ can then be defined from Eq. (2). Dividing $F_{\text {vib }}$ by $F_{\text {vis }}$, we formulate a nondimensional acoustothermal atomization parameter $A^{*}$ :

$$
A^{*}=\frac{F_{\mathrm{vib}}}{F_{\mathrm{vis}}}=K \sqrt{\frac{2 \pi \mu}{\rho^{3}}} \frac{m_{i}}{k_{B} T_{0}} a f^{3 / 2},
$$

where $K$ is a nondimensional scaling prefactor (here $K=6.268 \times 10^{4}$ ). Physically, increasing $A^{*}$ can be interpreted as increasing the applied acoustic energy.

In Fig. 5(a), we plot for all our simulations the maximum temperature rise $\left(\Delta T_{f}^{*}=\Delta T_{f} / T_{0}\right)$, a measure of heat flux $\left(q_{f}\right)$, against $A^{*}$. Figure 5(a) can be interpreted as an acoustothermal boiling curve; despite the inherent noise in NEMD temperature measurements, $A^{*}$ provides a reasonable classification of the three regimes reported here, tracing the rise in $q_{f}$ as evaporation gives way to nucleate boiling, and then the subsequent drop below $q_{f \text {,max }}$ coinciding with the onset of film boiling. In Fig. 5(b), we plot the nonevaporating layer thickness $\left(h_{\infty}\right)$ against $A^{*}$. A noteworthy outcome of this analysis is that $A^{*}$ provides an excellent estimate of $h_{\infty}$; a universal relation $h_{\infty} \propto-A^{*}$ is obtained, valid across all three regimes. We also observe that all water molecules are atomized once $A^{*}>A_{\mathrm{cr}}^{*}$ (here $A_{\mathrm{cr}}^{*}=1.1$ ).

Given their fundamental nature, the results presented in Fig. 5 have broad areas of application. First, we observe film evaporation for $A^{*}<0.45$, and our measurements show that this is accompanied by film superheating. Such fine control of thin-film evaporation has applications to evaporative self-assembly [47], while superheating can enhance reaction rates in lab-on-a-chip devices [48]. Second, we see that nucleate boiling can be achieved for $0.45<A^{*}<0.9$; the heat fluxes and film superheat observed are close to the theoretical and experimental 
limits, respectively, and higher than that possible by alternative methods. Enhanced nucleate boiling is relevant to many industries, including power generation, refrigeration, and desalination [49-51]. Third, film boiling is observed for $A^{*}>0.9$ and all film molecules are atomized if $A^{*}>A_{\mathrm{cr}}^{*}$, which is useful for drying applications [14]. Also, the flow of water over thin gas nanofilms entrapped on a surface can produce large slip, which has application to self-cleaning surfaces or drag-reducing or antifouling marine coatings [52]; inducing acoustothermal film boiling (i.e. $A^{*}>A_{\mathrm{cr}}^{*}$ ) on specialized surfaces could be a novel way to generate such gas nanofilms. Finally, our presented findings could spur more research into the physics of highfrequency acoustic streaming within the Stokes boundary layer, and eventually enable the incorporation of acoustothermal effects into continuum fluid models.

All data within this publication can be freely accessed in Ref. [53].

The authors thank the UK's Engineering and Physical Sciences Research Council (EPSRC) for support under Grants No. EP/N016602/1 and No. EP/R007438/1, and the ARCHER UK National Supercomputing Service. J. M. R. acknowledges the support of the Royal Academy of Engineering under the Chair in Emerging Technologies scheme.

*Corresponding author.

r.pillai@ed.ac.uk

[1] R. W. Wood and A. L. Loomis, Philos. Mag. 4, 417 (1927).

[2] R. J. Lang, J. Acoust. Soc. Am. 34, 6 (1962).

[3] R. L. Peskin and R. J. Raco, J. Acoust. Soc. Am. 35, 1378 (1963).

[4] S. Muñoz-Aguirre, T. Nakamoto, and T. Moriizumi, Sensors and Actuators, B: Chemical 105, 144 (2005).

[5] Z. J. Xu, R. Q. Chu, G. R. Li, X. Shao, and Q. R. Yin, Mater. Sci. Eng. B 117, 113 (2005).

[6] B. W. Mwakikunga, Crit. Rev. Solid State Mater. Sci. 39, 46 (2014).

[7] M. N. Topp, J. Aerosol Sci. 4, 17 (1973).

[8] F. Barreras, H. Amaveda, and A. Lozano, Exp. Fluids 33, 405 (2002).

[9] B. Avvaru, M. N. Patil, P. R. Gogate, and A. B. Pandit, Ultrasonics 44, 146 (2006).

[10] R. Rajan and A. B. Pandit, Ultrasonics 39, 235 (2001).

[11] S. Nii, in Ultrasonic Atomization, edited by $M$. Ashokkumar, F. Cavalieri, F. Chemat, K. Okitsu, A. Sambandam, K. Yasui, and B. Zisu, Handbook of Ultrasonics and Sonochemistry (Springer, Singapore, 2016), pp. 239-257.

[12] S. Kentish and M. Ashokkumar, in The Physical and Chemical Effects of Ultrasound, edited by H. Feng, G. Barbosa-Canovas, and J. Weiss, Ultrasound Technologies for Food and Bioprocessing (Springer, New York, 2011), pp. 1-12.

[13] O. Manor, A. R. Rezk, J. R. Friend, and L. Y. Yeo, Phys. Rev. E 91, 053015 (2015).
[14] C. Peng, S. Ravi, V. K. Patel, A. M. Momen, and S. Moghaddam, Energy 125, 498 (2017).

[15] While current atomization studies typically use frequencies in the $\mathrm{MHz}-\mathrm{GHz}$ range [16], in principle much higher $\mathrm{GHz}-\mathrm{THz}$ range bulk or surface vibrations are realizable [17-20].

[16] L. Y. Yeo and J. R. Friend, Annu. Rev. Fluid Mech. 46, 379 (2014).

[17] J. D. Choi, T. Feurer, M. Yamaguchi, B. Paxton, and K. A. Nelson, Appl. Phys. Lett. 87, 081907 (2005).

[18] Y. Ivry, N. Wang, and C. Durkan, Appl. Phys. Lett. 104, 133505 (2014).

[19] R. J. Shilton, M. Travagliati, F. Beltram, and M. Cecchini, Adv. Mater. 26, 4941 (2014).

[20] O. Matsuda and O.B. Wright, in Frontiers in Optical Methods (Springer, New York, 2014), pp. 129-151.

[21] J. Kondoh, N. Shimizu, Y. Matsui, and S. Shiokawa, IEEE Trans. Ultrason. Ferroelectr. Freq. Control 52, 1881 (2005).

[22] J. Kondoh, N. Shimizu, Y. Matsui, M. Sugimoto, and S. Shiokawa, Sensors and Actuators, A: Physical 149, 292 (2009).

[23] T. Roux-Marchand, D. Beyssen, F. Sarry, and O. Elmazria, IEEE Trans. Ultrason. Ferroelectr. Freq. Control 62, 729 (2015).

[24] R. J. Shilton, V. Mattoli, M. Travagliati, M. Agostini, A. Desii, F. Beltram, and M. Cecchini, Adv. Funct. Mater. 25, 5895 (2015).

[25] H. Li, Y. Li, and Z. Li, Ultrason. Sonochem. 4, 217 (1997).

[26] F. Tingaud, S. Ferrouillat, S. Colasson, A. Bontemps, and O. Bulliard-Sauret, Ultrason. Sonochem. 20, 1046 (2013).

[27] See Supplemental Material at http://link.aps.org/ supplemental/10.1103/PhysRevLett.121.104502 for information about the model details, simulation setup, and post-simulation measurement of relevant parameters.

[28] J. L. Abascal and C. Vega, J. Chem. Phys. 123, 234505 (2005).

[29] J. P. Ryckaert, G. Ciccotti, and H. J. Berendsen, J. Comput. Phys. 23, 327 (1977).

[30] S. Plimpton, J. Comput. Phys. 117, 1 (1995).

[31] J. Zhang, M. K. Borg, K. Sefiane, and J. M. Reese, Phys. Rev. E 92, 1 (2015).

[32] J. Zhang, M. K. Borg, K. Ritos, and J. M. Reese, Langmuir 32, 1542 (2016).

[33] M. S. Plesset and A. Prosperetti, Annu. Rev. Fluid Mech. 9, 145 (1977).

[34] S. Balibar and F. Caupin, J. Phys. Condens. Matter 15, S75 (2003).

[35] R. Zhang, Y. Ikoma, and T. Motooka, Nanotechnology 21, 105706 (2010).

[36] S. C. Maroo and J. Chung, Nanoscale Res. Lett. 6, 72 (2011).

[37] A. Hens, R. Agarwal, and G. Biswas, Int. J. Heat Mass Transfer 71, 303 (2014).

[38] G. Nagayama, M. Kawagoe, A. Tokunaga, and T. Tsuruta, Int. J. Thermal Sciences 49, 59 (2010).

[39] T. Yamamoto and M. Matsumoto, J. Therm. Sci. Technol. 7, 334 (2012). 
[40] S. C. Maroo and J. N. Chung, J. Heat Transfer 135, 061501 (2013).

[41] B. Peng, W. He, X. Hao, Y. Chen, and Y. Liu, Comput. Mater. Sci. 87, 260 (2014).

[42] W. R. Gambill and J. H. Lienhard, J. Heat Transfer 111, 815 (1989).

[43] M. Legay, N. Gondrexon, S. Le Person, P. Boldo, and A. Bontemps, Inter. J. Chem. Engin. 2011, 670108 (2011).

[44] J. Yu and H. Wang, Int. J. Heat Mass Transfer 55, 1218 (2012).

[45] V. P. Carey and A. P. Wemhoff, J. Heat Transf. 128, 1276 (2006).

[46] X. Yang and Y. Y. Yan, Appl. Therm. Eng. 31, 640 (2011).
[47] J. L. Plawsky, A. G. Fedorov, S. V. Garimella, H. B. Ma, S. C. Maroo, L. Chen, and Y. Nam, Nanoscale Micro. Thermophys. Eng. 18, 251 (2014).

[48] J. Reboud, Y. Bourquin, R. Wilson, G. S. Pall, M. Jiwaji, A. R. Pitt, A. Graham, A. P. Waters, and J. M. Cooper, Proc. Natl. Acad. Sci. U.S.A. 109, 15162 (2012).

[49] N. A. Patankar, Soft Matter 6, 1613 (2010).

[50] H. M. Kwon, J. C. Bird, and K. K. Varanasi, Appl. Phys. Lett. 103, 201601 (2013).

[51] N. S. Dhillon, J. Buongiorno, and K. K. Varanasi, Nat. Commun. 6, 8247 (2015).

[52] S. B. Ramisetti, M. K. Borg, D. A. Lockerby, and J. M. Reese, Phys. Rev. Fluids 2, 084003 (2017).

[53] http://dx.doi.org/10.7488/ds/2415. 